\title{
New-Onset Ventricular Arrhythmias in Patients with Left Ventricular Dysfunction after Coronary Surgery: Incidence, Risk Factors, and Prognosis
}

\author{
Nan Cheng, MD, Changqing Gao, MD, Rong Wang, MD, Ming Yang, MD, Lin Zhang, MD \\ Department of Cardiovascular Surgery, Chinese PLA General Hospital, Beijing, China
}

\section{ABSTRACT}

Background: The incidence, risk factors, and long-term prognosis of new-onset ventricular tachycardia (VT) and ventricular fibrillation (VF) after coronary artery bypass graft surgery $(\mathrm{CABG})$ in patients with impaired left ventricular function have not been thoroughly examined.

Methods: This study enrolled 612 consecutive patients with impaired left ventricular function (ejection fraction $<50 \%$ ) undergoing CABG at a single institution between March, 1996, and September, 2015. Outcomes were analyzed and compared, including in-hospital mortality and long-term survival. After a propensity-score, matching was performed to adjust for differences between the two cohorts. Factors significantly associated with VT/VF were also investigated using multivariate logistic regression.

Results: Of the 600 patients included in the analyses, 92 (15.3\%; 95\% confidence interval [CI] 12.5-18.3\%) had new-onset VT/VF postoperatively. Before propensity matching, patients with postoperative VT/VF were more likely to have renal failure, intra-aortic balloon pump support, lower preoperative ejection fraction (EF), and a larger left ventricle than those without VT/VF. Multivariate regression identified three preoperative risk factors and one protective factor that were independently associated with new-onset VT/VF: previous renal failure (odds ratio [OR] 4.42, $P=.02$ ), left ventricular end-diastolic dimension enlargement (OR 1.83, $P=.03$ ), ejection fraction (OR $1.88, P=.02$ for $\mathrm{EF} \geq 30$ and $<40 \%$ versus $\geq 40 \%$ and $<50 \%$; OR $5.46, P=.00$ for $\mathrm{EF}<30 \%$ versus $\geq 40 \%$ and $<50 \%$ ), and preoperative $\beta$-blockers (OR $0.58, P=.03)$. The median follow-up time was 46.6 months. In the propensity-matched cohorts, survival for patients who had in-hospital VT/VF was lower than that of the non-VT/ VF group (89.9\% versus $97.6 \% ; P<.05)$.

Conclusion: This study shows a high incidence of newonset VT/VF after CABG in patients with impaired left ventricular function. The early and long-term survival rates were significantly lower in the VT/VF group. Preoperative renal failure, left ventricular end-systolic dimension enlargement,

Received November 26, 2017; accepted December 9, 2017.

Correspondence: Rong Wang, MD, Department of Cardiovascular Surgery, the PLA General Hospital, No.28. Fuxing Road, Haidian District, Beijing, China, 100853; +86-10-66938135 (e-mail: wangrongd@126.com), and Changqing Gao, MD, Department of Cardiovascular Surgery, the PLA General Hospital, No.28. Fuxing Road, Haidian District, Beijing, China, 100853; +86-1066938035 (e-mail: gaochq301@gmail.com). and the severity of left ventricular function were independently associated with the development of new-onset VT/VF after CABG surgery. Preoperative use of beta-blocker was proved to be protective in reducing both $\mathrm{VT} / \mathrm{VF}$ incidence and in-hospital mortality in CABG patients with impaired left ventricular function following CABG. When considering these data, a prescription of beta-blockers is prognostically indicated to CABG patients, especially those with new-onset VT/NF postoperatively.

\section{BACKGROUND}

Though coronary artery bypass grafting (CABG) can reduce the incidence of sudden cardiac death in selected patients, newonset ventricular arrhythmia after CABG has been shown to be a lethal complication [Mouws 2017; Yeung-Lai-Wah 2004; Smith 1992]. Despite the fact that left ventricular ejection fraction (LVEF) has been proved to be the strongest risk factor for new-onset postoperative sustained ventricular tachycardia (VT) and ventricular fibrillation (VF) [Ascione 2004; Bigger 1984; Bonato 2016], the incidence and prognostic importance of ventricular dysrhythmias in patients with poor left ventricular function after surgical revascularization remains unknown. Many cardiac diseases, such as acute ischemia and previous myocardial infarction (MI), may increase the risk of ventricular arrhythmias, as previously reported [Mouws 2017; Chen 2015; El-Chami 2012; Ducceschi 2000]. These studies, however, were based mostly on patients with normal left ventricular function preoperatively. The CABG Patch trial has included patients who had ischemic dilated cardiomyopathy with LVEF $<36 \%$, and of the 198 deaths in this trial, up to $22 \%$ were due to ventricular arrhythmias (including primary and secondary causes of death). However, the risk factors present in most of these patients were unclear [Bigger 1984].

The aim of this study was to identify the clinical predictors of ventricular arrhythmias (VT and VF) in patients with impaired left ventricular function $(<50 \%)$ after CABG, investigate the associations between patient and operative factors and the occurrence of VT/VF, and describe the impact of $\mathrm{VT} / \mathrm{VF}$ on early and late mortality in this unique group of patients undergoing myocardial revascularization.

\section{METHODS}

\section{Patient Selection}

A nested case-control study was carried out using data collected prospectively from consecutive patients undergoing non-emergent CABG. All patients signed an informed 
Table 1. Baseline Characteristics Comparing Patients with VT/VF to Those without VT/VF

\begin{tabular}{|c|c|c|c|c|c|c|}
\hline Gender: Male & $81(88.0)$ & $442(88.7)$ & 0.785 & $73(88.0)$ & $75(90.4)$ & 0.617 \\
\hline \multicolumn{7}{|l|}{ Age (yrs) } \\
\hline$>55$ and $\leq 70$ & $47(51.1)$ & $297(58.8)$ & & $43(51.8)$ & $44(53.0)$ & \\
\hline$>70$ & $26(28.3)$ & $106(20.9)$ & & $21(25.3)$ & $21(25.3)$ & \\
\hline \multicolumn{7}{|l|}{ NYHA class } \\
\hline 1 & $6(6.5)$ & $23(4.5)$ & 0.225 & $5(6.0)$ & $6(7.2)$ & 0.966 \\
\hline ॥ & $40(43.5)$ & $248(48.8)$ & & $40(48.2)$ & $37(44.6)$ & \\
\hline Diabetes & $34(37.0)$ & $146(28.7)$ & 0.114 & $31(37.3)$ & $29(34.9)$ & 0.747 \\
\hline Dyslipidemia & $21(22.8)$ & $80(15.7)$ & 0.095 & $16(19.3)$ & $18(21.7)$ & 0.701 \\
\hline Hypertension & $47(51.1)$ & $268(52.8)$ & 0.768 & $42(50.6)$ & $46(55.4)$ & 0.534 \\
\hline Current smoker & $48(52.2)$ & $270(53.1)$ & 0.863 & $44(53.0)$ & $45(54.2)$ & 0.876 \\
\hline Previous Cerebrovascular accident & $8(8.7)$ & $75(14.8)$ & 0.121 & $7(8.4)$ & $8(9.6)$ & 0.787 \\
\hline Respiratory disease & $4(4.3)$ & $18(3.5)$ & 0.706 & $2(2.4)$ & $2(2.4)$ & 1.000 \\
\hline Renal failure & $6(6.6)$ & $8(1.6)$ & 0.004 & $3(3.6)$ & $6(7.2)$ & 0.304 \\
\hline Peripheral arterial disease & $4(4.3)$ & $12(2.4)$ & 0.277 & $2(2.4)$ & $4(4.8)$ & 0.406 \\
\hline LA enlargement & $68(73.9)$ & $375(73.8)$ & 0.985 & $61(73.5)$ & $56(67.5)$ & 0.395 \\
\hline LV enlargement & $56(60.9)$ & $238(46.9)$ & 0.013 & $50(60.2)$ & $50(60.2)$ & 1.000 \\
\hline Ass. MVR & $7(7.6)$ & $26(5.1)$ & 0.335 & $4(4.8)$ & $7(8.4)$ & 0.349 \\
\hline Ass. AVR & $3(3.3)$ & $13(2.6)$ & 0.701 & $2(2.4)$ & $2(2.4)$ & 1.000 \\
\hline Ass. LVA & $10(10.9)$ & $74(14.6)$ & 0.347 & $10(12.0)$ & $10(12.0)$ & 1.000 \\
\hline Calcium-antagonist & $11(12.0)$ & $35(6.9)$ & 0.093 & $8(9.6)$ & $14(16.9)$ & 0.170 \\
\hline Beta-blockers & $64(69.6)$ & $317(62.4)$ & 0.073 & $50(60.2)$ & $47(56.6)$ & 0.637 \\
\hline ACE-inhibitor & $71(82.6)$ & $437(85.0)$ & 0.344 & $46(55.4)$ & $49(60.1)$ & 0.221 \\
\hline
\end{tabular}

LVA indicates left ventricular aneurysmectomy; NYHA, New York Heart Association; MI, myocardial infarction; LM, left main; IABP, intra-aortic balloon pump; EF, ejection fraction; LA, left atrium; LV, left ventricular; MVR, mitral valve replacement; AVR, aortic valve replacement; VT/VF, ventricular tachycardia/ventricular fibrillation.

consent form approved by the Institutional Review Board. Each patient's preoperative clinical data were collected, and their perioperative data recorded. The analysis included all patients undergoing CABG between March 1, 1996, and September 15, 2015, with impaired left ventricular function (EF $<50 \%$ ) and no previous history of VT/VF. The surgeons in our 
Table 2. Multivariate Analysis for Risk Factors of Postoperative VT/VF

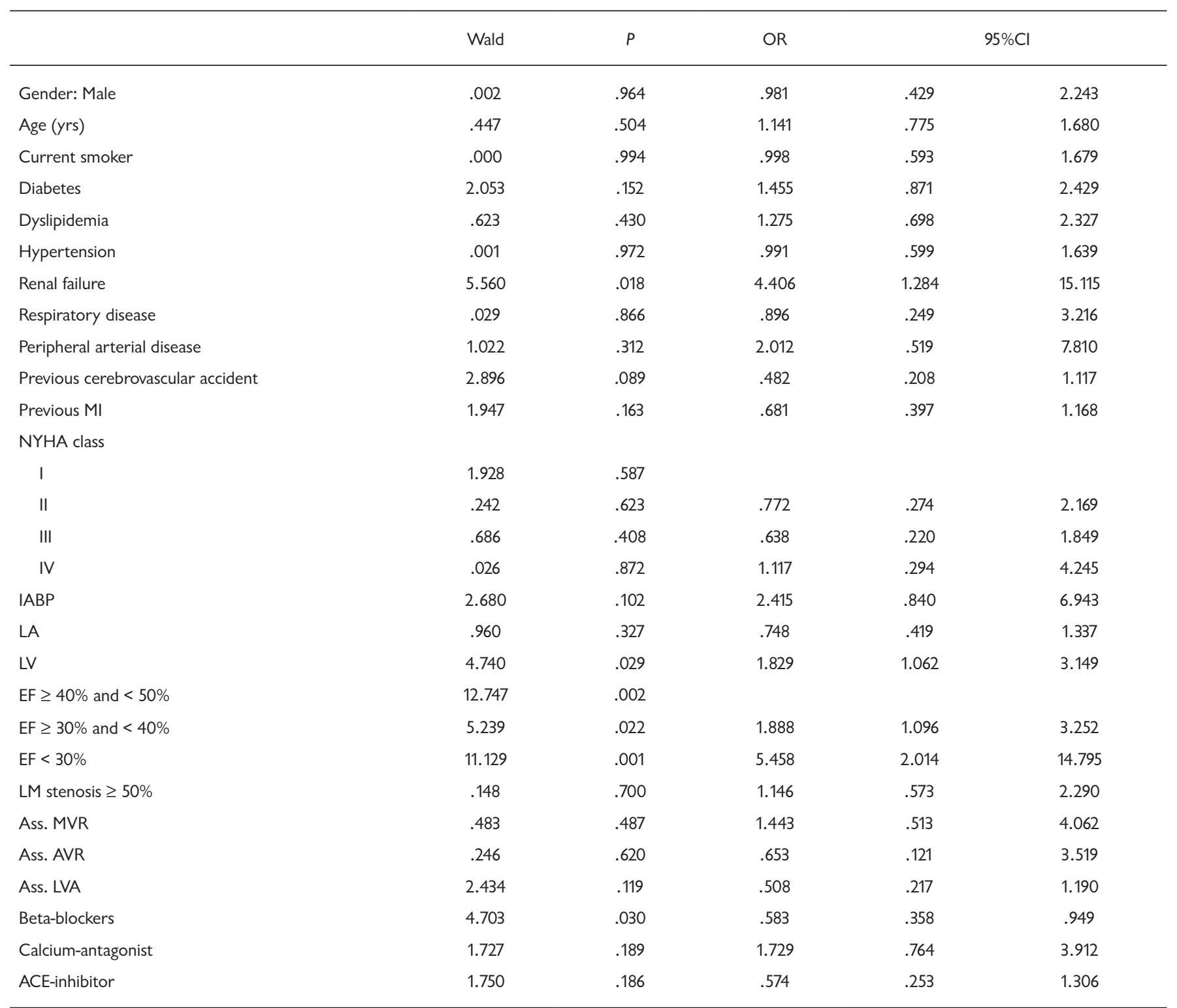

$\mathrm{Cl}$ indicates confidence interval; OR, odds ratio; NYHA, New York Heart Association; MI, myocardial infarction; LM, left main; IABP, intra-aortic balloon pump;

$E F$, ejection fraction; LA, left atrium; LV, left ventricular; MVR, mitral valve replacement; AVR, aortic valve replacement.

institution carried out on-pump CABG as their routine technique. Patients who underwent concomitant interventions such as valvar replacement or left ventricular aneurysmectomy were deliberately included to examine whether associated surgical procedures could increase ventricular susceptibility to arrhythmias. Beta-blocker, calcium-antagonist, and ACEinhibitor were used as guideline-directed medical therapy for heart failure with reduced ejection fraction. A greater proportion of patients also received beta-blockers for supraventricular arrhythmias or sinus tachycardia and hypertension.

During the study, we defined cases as patients who required advanced cardiac life support interventions to terminate VT/
VF and to stabilize the hemodynamic status postoperatively before discharge from the hospital. All other patients were defined as controls. The preoperative characteristics are summarized in Table 1.

\section{Operative Techniques}

All patients were premedicated with morphine and diazepam. The primary anesthetics included isoflurane and fentanyl. Ventilation was performed to maintain $\mathrm{PCO} 2$ at 35-45 $\mathrm{mmHg}$ and $\mathrm{PO}_{2}>80 \mathrm{mmHg}$. The blood pressure, central venous pressure, and pulmonary artery pressure were continuously monitored by radial artery catheter and pulmonary 
Table 3. Outcomes of Propensity-Matched Analysis

\begin{tabular}{|c|c|c|c|c|}
\hline Outcomes & $\mathrm{VT} / \mathrm{VF}(\mathrm{n}=83)$ & No VT/VF (n=83) & $\chi^{2} / \mathrm{t}$ & $P$ \\
\hline ICU Stay (days) & $7.03 \pm 6.77$ & $4.82 \pm 2.39$ & 2.806 & .006 \\
\hline Reintubation & $13(15.7 \%)$ & $0(0 \%)$ & 14.105 & .000 \\
\hline Postoperative renal failure requiring hemofiltration & $6(7.2 \%)$ & $2(2.4 \%)$ & 2.101 & .147 \\
\hline Postoperative myocardial infarction & $1(1.2 \%)$ & $0(0 \%)$ & 1.006 & .316 \\
\hline Death & $7(8.4 \%)$ & $0(0 \%)$ & 7.308 & .007 \\
\hline
\end{tabular}

ICU indicates intensive care unit; VT/VF, ventricular tachycardia/ventricular fibrillation; IABP, intra-aortic balloon pump.

arterial catheter. A seven-lead electrocardiogram (ECG) was routinely employed. Cardiopulmonary bypass was performed using a membrane oxygenator, hemodilution, moderate systemic hypothermia $\left(26-28^{\circ} \mathrm{C}\right)$, and HTK solution cardioplegia. The revascularization strategy included performing a left anterior descending graft by internal thoracic artery, followed by saphenous vein grafts to other target vessels. The distal anastomoses of saphenous vein grafts were performed under aortic cross-clamp, followed by proximal vein grafts with partial aortic occlusion or single cross-clamp technique depending on the surgeons' preference.

\section{Postoperative Management}

Patients were transferred to the intensive care unit (ICU) after the operation. Inotropic support was used to maintain systolic blood pressure $>100 \mathrm{mmHg}$ and a cardiac index $>2.3 \mathrm{~L} / \mathrm{min} / \mathrm{m}^{2}$. Patients were extubated as they met the following criteria: hemodynamic stability, no major bleeding $(50 \mathrm{~mL} / \mathrm{h})$, consciousness recovery, and normothermia. Potassium and magnesium were maintained within the normal range. Twelve-lead ECGs were monitored daily postoperatively until the patients were transferred out of the ICU.

\section{Definitions}

Malignant VT/VF was defined as monomorphic or polymorphic ventricular tachycardia, with an episode of consecutive ventricular beats of $\geq 100$ per minute or ventricular fibrillation lasting more than 30s, unless terminated earlier by advanced cardiac life support because of hemodynamic collapse.

Management of VT/VF aimed to achieve assessment and treatment of reversible or correctable causes of arrhythmia, including metabolic disorders, myocardial ischemia or infarction, and potentially, proarrhythmic medications.

To reduce the influence of preoperative factors on comparison of the VT/VF and non-VT/VF groups, we used propensity score matching, which can balance the covariates of the two groups and thus reduce bias. A total of 22 demographics and preoperative risk factors were included, and a one to one propensity score match was obtained in every case, to the nearest score within $\pm 1 \%$ difference. Matched groups were compared to ensure that patient factors were matched adequately, defined as standardized deviations (SD) $<10 \%$ for all continuous and categorical variables.

\section{Follow-up}

The patients were continuously monitored until there was no presence of unstable hemodynamic status and they were transferred from the ICU. Patients with postoperative VT/ VF were treated with antiarrhythmic drugs, generally a combination of amiodarone and $\beta$-blockers. The early outcome (30 days postoperatively) and long term mortality (over 24 months) were recorded. Patients were followed up with by telephone and mailing.

\section{Statistical Analysis}

Continuous variables were expressed as mean \pm SD. Differences in clinical variables were tested by chi-square or Fisher exact tests for categorical variables, and the unpaired Student $t$ test for continuous variables. Conditional logistic regression was used to determine independent factors of postoperative VT/VF. All-cause mortality comparison was performed by Kaplan-Meier analysis (log-rank test) on the matched groups. We used proportional hazards Cox regression for the sevenyear follow-up to estimate the corresponding risk-adjusted hazard ratio (HR) (with $95 \%$ confidence interval [CI]) relating the two groups. A two-sided $P$ value of $<.05$ was regarded significant. Statistical analysis was performed with SPSS software (version 22; IBM, Armonk, NY, USA).

\section{RESULTS}

\section{Incidence of VT/VF}

During the study, a total of 612 patients with impaired left ventricular function $(\mathrm{EF}<50 \%)$ underwent $\mathrm{CABG}$. However, information from 12 patients on postoperative VT/VF was missing $(1.9 \%)$, and these patients were not included further. The data of 23 preoperative patient factors were completed for the remaining 600 cases, of which 528 (88.0\%) had 
Table 4. Multivariate Analysis of in-hospital mortality

\begin{tabular}{lccccc}
\hline & Wald & $P$ & OR & \multicolumn{2}{c}{$95 \% \mathrm{Cl}$} \\
Renal failure & 4.774 & .029 & 9.252 & 1.258 & 68.077 \\
EF & .259 & .611 & .703 & .181 & 2.732 \\
VF & 10.682 & .001 & 38.912 & 4.330 & 349.650 \\
Beta-Blocker & 4.581 & .032 & .150 & .026 & .852 \\
LV & 1.459 & .227 & 2.998 & .505 & 17.804 \\
\hline
\end{tabular}

$\mathrm{Cl}$ indicates confidence interval; OR, odds ratio; $\mathrm{EF}$, ejection fraction; LV, left ventricular; VF, ventricular fibrillation.

on-pump surgery. Ninety-two of the 600 patients $(15.3 \%$; $95 \%$ CI $12.5 \%-18.3 \%$ ) had new-onset VT/VF postoperatively. Seven patients died in the hospital or within 30 days of CABG (1.2\%), all of whom suffered VT/NF. No intraoperative occurrence of VT/NF was observed during the study.

\section{Propensity Score Matching Results}

Before propensity matching, patients with postoperative VT/VF were more likely to have renal failure (creatine $>200$ $\mu \mathrm{mol} / \mathrm{L}$ ), more intra-aortic balloon pump (IABP) support, lower preoperative EF, and a larger left ventricle than those without VT/VF (Table 1). Matched cohorts of 83 patients with and without VT/VF are also presented in Table 1, in which there were no significant differences between the groups with regard to all 23 preoperative factors.

\section{Risk factors and Clinical Outcomes of VT/VF}

Multiple logistic regression modeling identified three preoperative risk factors and one protective factor that were independently associated with new-onset VT/VF (Table 2): previous renal failure (OR 4.42, 95\% CI 1.27-15.26; $P=.02$ ), left ventricular end-systolic dimension enlargement (OR $1.83,95 \%$ CI 1.06-3.15; $P=.03$ ), LVEF (OR 1.88, $P=.02$ for $\mathrm{EF} \geq 30$ and $<40 \%$ versus $\geq 40 \%$ and $<50 \%$; OR $5.46, P=.00$ for $\mathrm{EF}<30 \%$ versus $\geq 40 \%$ and $<50 \%$ ), and preoperative $\beta$-blockers (OR 0.58, 95\% CI 0.36-0.95; $P=.03$ ).

Clinical outcome results for new-onset VT/VF and propensity-matched groups of 83 patients are displayed in Table 3. Patients with postoperative VT/VF had longer ventilation time $(57.70 \pm 86.90$ versus $32.40 \pm 37.65 \mathrm{~h}, P=.02)$ and ICU stay $(7.03 \pm 6.77$ versus $4.82 \pm 2.39$ days, $P=.01)$, more reintubation $(13[15.7 \%]$ versus $0, P=.00)$, and more postoperative strokes (7 [8.4\%] versus $1[1.2 \%], P=.03)$.

Multivariate analysis of predictors for in-hospital mortality, including VT/VF and other potential risk factors, showed that VT/NF (OR 38.91, 95\% CI 4.33-349.65; $P<.01$ ), previous renal failure (OR 9.25, 95\% CI 1.26-68.08; $P=.03$ ), and preoperative $\beta$-blockers (OR $0.15,95 \%$ CI $0.03-0.85$; $P=.03$ ) (Table 4).

\section{Follow-up}

The overall median time of follow-up was 46.6 months (interquartile range 28.0-71.6 months). Long-term survival for

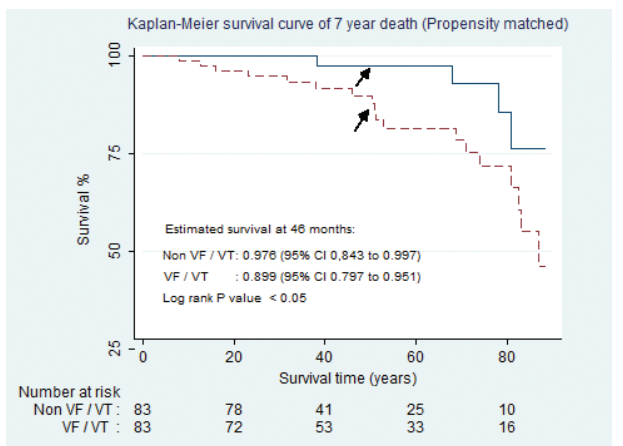

Kaplan-Meier curve that depicts the overall mortality between those with VT/VF and those without VT/VF at 7 year in the propensity matched cohort. VT indicates ventricular tachycardia; VF, ventricular fibrillation.

patients who had in-hospital VT/VF was lower than that of the non-VT/VF group $(89.9 \%$ versus $97.6 \% ; P<.05)$ (Figure).

\section{DISCUSSION}

The present cohort is one of the largest to be examined for risk factors in early and late outcomes of new-onset VT/VF after CABG surgery, in patients with impaired left ventricular function. The following observations were made from our study:

(1) New-onset VT/VF after CABG in patients with impaired LV function occurred more frequently than previously reported [Ascione 2003], with an incidence of $15.3 \%$.

(2) A significant independent relationship was found between the development of VT/VF and preoperative renal failure, left ventricular end-systolic dimension enlargement, and the severity of left ventricular function.

(3) Propensity score matching showed an increase of postoperative complications and higher early in-hospital mortality rate in patients who developed VT/VF. Multivariate analysis proved that $\mathrm{VT} / \mathrm{VF}$ was a strong predictor of early in-hospital mortality. Moreover, the long-term survival rate was also lower in the VT/VF group.

(4) $\beta$-Blockers were shown to have a favorable role not only in reducing arrhythmic death, but also in the reduction of overall mortality in high risk patients. Similar benefits were identified in previous studies of $\beta$-blocker use for the effects of antiarrhythmia [Ascione 2004; Brembilla-Perrot 2003].

\section{Incidence of New-Onset VT/VF}

In previous studies, a much lower incidence $(0.8-3.1 \%$ compared with $15.3 \%$ in the present study) of ventricular arrhythmias was found after CABG surgery [Ascione 2004; Brembilla-Perrot 2003; Steinberg 1999]. There are, however, important cohort differences between our series and those of the earlier reports:

(1) All of our patients had impaired left ventricular function $(<50 \%)$, whereas in previous studies, less than $30 \%$ of patients had abnormal left ventricular function [Ducceschi 2000; Steinberg 1999].

(2) The rate of prior MI in our cohort was $70 \%$, much higher than reported in earlier groups $(40-50 \%$ of patients 
having previous MI) [Chen 2015; Ducceschi 2000].

(3) About one-fifth of our patients (22\%) had concomitant surgeries (mitral valve replacement, aortic valve replacement, and left ventricular aneurysmectomy), compared with only $7 \%$ of patients in previous studies who had associated surgeries [Ducceschi 2000].

\section{Risk Factors}

In this study, we were able to examine some potential factors contributing to postoperative VT. Bigger et al have highlighted a negative prognostic outcome when VT/VF occurred in the setting of healing MI (more than seven days after MI) [Bigger 1984]. Cheema et al also demonstrated poor prognostic effects of VT occurring in the setting of acute MI (within the first days) [Cheema 1998]. However, from our multivariate analysis results, preoperative risk factors such as demographic characteristics or previous MI were not related to the occurrence of postoperative VT, contrary to previous reports [Pires 2001; Newby 1998]. This might be due to the fact that most of our pre-infarcted patients were operated on at least four weeks after acute MI.

As expected, and as reported by others, patients in our study, who had developed poor left ventricular function prior to surgery, remained susceptible to VT/VF despite a successful surgical revascularization procedure [El-Chami 2012; Ascione 2004; Azar 1997]. This may result from the residual effects of a previous ischemic event or prior infarcted scarring, which leads to arrhythmogenic foci and reentry pathways[Tam 1991]. These pathological changes constitute a stable arrhythmia substrate, which could not be reversed by surgical revascularization, and acts as a trigger for ventricular arrhythmias after a revascularization procedure [Tam 1991]. In addition to this, prior MI scarring and areas of myocardial necrosis contribute to left ventricular remodeling and dilation, which in turn aggravate the left ventricular dysfunction and myocardial ischemia. Thus, it is reasonable to assume that patients with poor left ventricular function have an exponentially increased tendency to develop sustained runs of ventricular arrhythmias.

Another incremental risk factor in ventricular arrhythmias is renal failure (creatine $>200 \mu \mathrm{mol} / \mathrm{L}$ ), which has been identified by previous research reporting that impaired renal function is also associated with left ventricular systolic dysfunction and ventricular arrhythmia [Mozos 2014; Bonato 2013]. Age, hemoglobin levels, several laboratory markers, and EF were factors associated with ventricular arrhythmia in patients with renal disease that were or were not undergoing hemodialysis or renal transplantation [Mozos 2014; Bonato 2013]. Oliveira and colleagues also suggested that ventricular arrhythmia was associated with poor clinical outcomes in nondialyzed patients with chronic kidney disease [Bonato 2016].

The present analysis demonstrates that beta-blocker use was associated with the reduction of both postoperative VT/ VF incidence and in-hospital mortality. It has been reported that recent cardiac surgery could increase the adrenergic tone and reduce vagal tone, especially in post-MI patients with a latent ventricular ectopic rhythm activity, which could facilitate the occurrence of VT/VF [Coumel 1993; Willich 1993].
Beta-blocker could act as a role in reducing sympathetic and parasympathetic tone [Brembilla-Perrot 2003]. Furthermore, the European Myocardial Infarct Amiodarone Trial (EMIAT) and the Canadian Amiodarone Myocardial Infarction Arrhythmia Trial(CAMIAT) have both demonstrated the beneficial effect of beta-blocker in managing patients with more rapid baseline heart rates [Cairns 1997; Julian 1997]. These findings explain the protective effects of beta-blocker in reducing arrhythmic mortality in post-MI patients with impaired left ventricular function.

\section{Prognosis}

In this study, patients with postoperative VT/VF that were discharged home appeared to have a lower late survival rate compared with the controls, contrary to some prior work that presented comparable long-term results between the VT/VF group and controls [El-Chami 2012; Ascione 2004]. Bigger et al have concluded that ventricular arrthymias may interact with impaired left ventricular function on late mortality after myocardial infarction [Bigger 1984]. The differences in longterm survival between our study and others may be attributed to the interaction of ventricular arrthymias and left ventricular dysfunction on the VT/VF group. In our study, another finding, revealed by the propensity score matched results, was a much higher incidence of stroke in the VT/VF group. This might be the watershed due to cerebral hypoperfusion.

Since multivariate analysis indicated that -blockers use was independently associated with improved survival and reduced incidence of VT/VF following cardiac surgery, it can be inferred that $\beta$-blockers could act as a protective role in reducing VT/VF recurrence and arrhythmic mortality in the long-term results, given the ominous prognosis and the tendency of recurrence of lethally ventricular arrhythmias after CABG. Based on these facts, it is appropriate to prescribe beta-blockers to CABG patients with severely impaired left ventricular function, especially those having new-onset VT/ VF postoperatively.

\section{Study Limitations}

The main limitation of our study is that despite the large number of patients with impaired left ventricular function, the data presented can only be applied to the patients according to our definitions. This is because our research was based on the observations on patients who experienced VT/VF within a four to seven day window in the ICU after surgery. Although patients who were transferred from the ICU or discharged from the hospital suffering VT/VF may also constitute this cohort, they were not included in this study. In addition, other risk factors that demonstrated no significance from a statistical perspective may nevertheless contribute a clinically important increase in risk (eg, preoperative IABP and MI). These potential risk factors of VT/VF need to be further investigated in larger cohorts. Despite these limitations, we believe that our study provides valuable information on the prediction and prognosis of those patients at high risk for postoperative ventricular arrhythmia. 
CONCLUSION

This study shows a high incidence of new-onset VT/VF after CABG in patients with impaired left ventricular function. The early and long-term survival rates were significantly lower in the VT/VF group. Preoperative renal failure, left ventricular end-systolic dimension enlargement, and the severity of left ventricular function were independently associated with the development of new-onset VT/VF after CABG surgery. Preoperative use of beta-blocker was proved protective in reducing both VT/VF incidence and in-hospital mortality in CABG patients with impaired left ventricular function following CABG. Based on these results, a prescription of betablockers is prognostically indicated to CABG patients, especially those having new-onset VT/VF postoperatively.

\section{REFERENCES}

Ascione R, Reeves BC, Santo K, et al. 2004. Predictors of new malignant ventricular arrhythmias after coronary surgery: a case-control study. J Am Coll Cardiol 43:1630-8.

Ascione R, Narayan P, Rogers CA, et al. 2003. Early and midterm clinical outcome in patients with severe left ventricular dysfunction undergoing coronary artery surgery. Ann Thorac Surg 76:793-9.

Azar RR, Berns E, Seecharran B, et al. 1997. De novo monomorphic and polymorphic ventricular tachycardia following coronary artery bypass grafting. Am J Cardiol 80:76-8.

Bigger JT, Jr., Fleiss JL, Kleiger R, et al. 1984. The relationships among ventricular arrhythmias, left ventricular dysfunction, and mortality in the 2 years after myocardial infarction. Circulation. 69:250-8.

Bonato FO, Watanabe R, Lemos MM, et al. 2016. Asymptomatic ventricular arrhythmia and clinical outcomes in chronic kidney disease: a pilot study. Cardiorenal Med 7:66-73.

Bonato FO, Lemos MM, Cassiolato JL, et al. 2013. Prevalence of ventricular arrhythmia and its associated factors in nondialyzed chronic kidney disease patients. PloS One 8:e66036.

Brembilla-Perrot B, Villemot JP, Carteaux JP, et al. 2003. Postoperative ventricular arrhythmias after cardiac surgery: immediate- and long-term significance Pacing Clin Electrophysiol 26:619-25.

Budeus M, Feindt P, Gams E, et al. 2006. Risk factors of ventricular tachyarrhythmias after coronary artery bypass grafting. Int J Cardiol 113:201-8.

Cairns JA, Connolly SJ, Roberts R, et al. 1997. Randomised trial of outcome after myocardial infarction in patients with frequent or repetitive ventricular premature depolarisations: CAMIAT. Canadian amiodarone myocardial infarction arrhythmia trial investigators. Lancet 349:675-82.
Cheema AN, Sheu K, Parker M, et al. 1998. Nonsustained ventricular tachycardia in the setting of acute myocardial infarction: tachycardia characteristics and their prognostic implications. Circulation 98:2030-6.

Chen JW, Lin CH, Hsu RB. 2015. Malignant ventricular arrhythmias after off-pump coronary artery bypass. J Formos Med Assoc 114:936-42.

Coumel P. 1993. Cardiac arrhythmias and the autonomic nervous system. J Cardiovasc Electrophysiol 4:338-55.

Ducceschi V, D'Andrea A, Liccardo B, et al. 2000. Ventricular tachyarrhythmias following coronary surgery: predisposing factors. Int J Cardiol 73:43-8.

El-Chami MF, Sawaya FJ, Kilgo P, et al. 2012. Ventricular arrhythmia after cardiac surgery: incidence, predictors, and outcomes. J Am Coll Cardiol 60:2664-71.

Julian DG, Camm AJ, Frangin G, et al. 1997. Randomised trial of effect of amiodarone on mortality in patients with left-ventricular dysfunction after recent myocardial infarction: EMIAT. European myocardial infarct amiodarone trial investigators. Lancet 349:667-74.

Mouws EM, Yaksh A, Knops P, et al. 2017. Early ventricular tachyarrhythmias after coronary artery bypass grafting surgery: Is it a real burden? J Cardiol 70:263-70

Mozos I. 2014. Laboratory markers of ventricular arrhythmia risk in renal failure. Biomed Res Int 2014:509204.

Newby KH, Thompson T, Stebbins A, et al. 1998. Sustained ventricular arrhythmias in patients receiving thrombolytic therapy: incidence and outcomes. The GUSTO Investigators. Circulation 98:2567-73.

Pires LA, Lehmann MH, Buxton AE, et al. 2001. Multicenter unsustained tachycardia trial I. Differences in inducibility and prognosis of inhospital versus out-of-hospital identified nonsustained ventricular tachycardia in patients with coronary artery disease: clinical and trial design implications. J Am Coll Cardiol 38:1156-62.

Smith RC, Leung JM, Keith FM, et al. 1992. Ventricular dysrhythmias in patients undergoing coronary artery bypass graft surgery: incidence, characteristics, and prognostic importance. Study of Perioperative Ischemia (SPI) Research Group. Am Heart J 123:73-81.

Steinberg JS, Gaur A, Sciacca R, et al. 1999. New-onset sustained ventricular tachycardia after cardiac surgery. Circulation 99:903-8.

Tam SK, Miller JM, Edmunds LH Jr. 1991. Unexpected, sustained ventricular tachyarrhythmia after cardiac operations. J Thorac Cardiovasc Surg 102:883-9.

Willich SN, Maclure M, Mittleman M, et al. 1993. Sudden cardiac death. Support for a role of triggering in causation. Circulation 87:1442-50

Yeung-Lai-Wah JA, Qi A, McNeill E, et al. 2004. New-onset sustained ventricular tachycardia and fibrillation early after cardiac operations. Ann Thorac Surg 77:2083-8. 